\title{
Second order differential equations with random perturbations and small parameters
}

\author{
Kamenskii, M., *Pergamenchtchikov, S. ${ }^{\dagger}$ and Quincampoix, M. ${ }^{\ddagger}$ \\ January 19, 2018
}

\begin{abstract}
We consider boundary value problems for differential equations of second order containing a brownian motion (random perturbation) and a small parameter. For this case we prove a special existence and unicity theorem for random solutions. The asymptotic behavior of these solutions as small parameter goes to zero is studied. The stochastic averaging theorem for such equations is shown. The limits in the explicit form for the solutions as a small parameter goes to zero are found.
\end{abstract}

Keywords: Boundary value problems, stochastic averaging method, Green functions.

AMS 2000 Subject Classifications: Primary: 60H10, 60J60, 58J37, 34B05, 34C29, 34D15, 34B27

*Voronezh State University, Universitetskay pl. 1, 394063 Voronezh, Russia, e-mail: Mikhailkamenski@mail.ru

†Laboratoire de Mathématiques Raphael Salem, CNRS - UMR 6085, Université de Rouen, France and International Laboratory of SSP\&QF, National Research Tomsk State University, Russia e-mail: Serge.Pergamenchtchikov@univ-rouen.fr

${ }_{\ddagger}^{\ddagger}$ Laboratoire de Mathématiques de Bretagne Atlantique, CNRS UMR 6205, Université de Bretagne Occidentale, 6 avenu Victor Le Gorgeu - CS 9383729238 BREST Cedex 3, FRANCE, e-mail : Marc.Quincampoix@univ-brest.fr 


\section{Introduction}

This paper is devoted to a limiting problem for the differential equations containing stochastic terms and small parameters. Such problems remount to the papers on the asymptotic theory for the ordinary differential equations. There exist two general methods for study these problems: the Bogolubov averaging principle [7] and the singularly perturbation Tikhonov method [23][24]. In the first case the quick variables have not limit, but in the second case the quick variables go to some limit. Usually, to find this limit one has to replace in the equations the small parameter by zero, and the solution of the limiting problem gives the limit for the quick variables. The situation becomes essentially difficult if we consider the boundary problems. So, let us consider, for example, the ordinary second order differential equation with the small parameter on the interval $[0,1]$, i.e.

$$
\ddot{x}^{\varepsilon}(t)=\varepsilon\left(p x^{\varepsilon}(t)+f(t)\right),
$$

where $p>0$. In this paper we consider the following boundary conditions: First Neumann boundary conditions

$$
x(0)=x(1)=0 .
$$

Second Neumann boundary conditions

$$
\dot{x}(0)=\dot{x}(1)=0 \text {. }
$$

Periodic boundary conditions

$$
x(0)=x(1) \quad \text { and } \quad \dot{x}(0)=\dot{x}(1) .
$$

Note that if we replace in the equation (1.1) the parameter $\varepsilon$ by zero, we obtain the following limit problem

$$
\ddot{x}=0 \text {. }
$$

It should be noted that this equation with the first boundary condition has unique solution $x=0$. However, for the boundary conditions (1.3) or (1.4) this limiting problem has not unique solution, since any constant satisfies this equation, i.e. in this case the limiting problem does not give any information about the asymptotic (as $\varepsilon \rightarrow 0$ ) behavior of the solution of the equation (1.1). Therefore, a natural question arises, how to calculate this constant. For 
the boundary conditions (1.3) or (1.4) through the Green functions method (see Remark 3.1 in Section 3) one can show, that for any $0<t<1$

$$
\lim _{\varepsilon \rightarrow 0} x^{\varepsilon}(t)=-\frac{1}{p} \int_{0}^{1} f(s) \mathrm{d} s .
$$

There exists a vast literature on the stochastic differential equations with small parameters and its applications (see, for example, [12], [13] and [17] for details and references therein). It turns out that the small parameter methods for stochastic differential equations are useful for the optimal stochastic control problems ([1], [2], [3]), for stochastic volatility financial markets ([5], [6], [11]), for the statistical estimation in multi-scale stochastic systems ([20], [21] [22]) and for many other applied problems. Usually, one considers initial or terminal conditions for the stochastic differential equations. In this paper we propose to extend both these problems, i.e. we introduce the random differential equation of the second order with the boundary conditions of the forms $(1.2)-(1.4)$. For such models we study the stochastic averaging principle. This problem is well known for the diffusion processes (for example, [12] or [16]). To understand the averaging principle problem we start with the following example. We consider on the interval $[0, \varepsilon]$ (for some small parameter $0<\varepsilon<1$ ) the linear stochastic differential equation of second order with quickly oscillating and "small" random force ("white noise"), i.e.

$$
\ddot{y}^{\varepsilon}(u)=p y^{\varepsilon}(u)+f(u / \varepsilon)+\delta(u / \varepsilon) \sqrt{\varepsilon} \dot{w}_{u},
$$

where $p>0$ is some fixed parameter and $\delta$ is a $\mathbb{R} \rightarrow \mathbb{R}$ square integrable function, i.e.

$$
\|\delta\|^{2}=\int_{0}^{1} \delta^{2}(t) \mathrm{d} t<\infty .
$$

Moreover, $\left(w_{t}\right)_{t \geq 0}$ is a standard Brownian motion and, therefore, $\left(\dot{w}_{t}\right)_{t \geq 0}$ is "white noise". By putting here $t=u / \varepsilon$ and $x^{\varepsilon}(t)=y^{\varepsilon}(t \varepsilon)$ we obtain on the time interval $[0,1]$ the following stochastic differential equation with small parameter

$$
\ddot{x}^{\varepsilon}(t)=\varepsilon^{2}\left(p x^{\varepsilon}(t)+f(t)\right)+\varepsilon^{2} \delta(t) \dot{W}_{t},
$$

where $W_{t}=w_{t \varepsilon} / \sqrt{\varepsilon}$ is a standard Brownian motion as well. In this paper we consider a more general nonlinear case, i.e. the equation of the following form

$$
\mathrm{d} \dot{x}^{\varepsilon}(t)=\varepsilon^{2}\left(A\left(t, x^{\varepsilon}(t)\right)+f(t)\right) \mathrm{d} t+\varepsilon^{2} \delta(t) \mathrm{d} W_{t},
$$


where $A$ is some $\mathbb{R}_{+} \times \mathbb{R} \rightarrow \mathbb{R}$ nonrandom function and $\left(W_{t}\right)_{0 \leq t \leq 1}$ is the standard Brownian motion.

The problem is to study the asymptotic (as $\varepsilon \rightarrow 0$ ) behavior of the equation (1.7). First of all we have to provide an existence and unicity theorem for solutions with the boundary conditions $(1.2)-(1.4)$. To this end we make use of a some uniform version of the implicit function theorem. Moreover, to study the asymptotic behavior we propose an averaging method based on the Green functions approach which is usually used for deterministic differential equations (see, for example, [4], [10, p. 31], [14] and the references therein).

The paper is organized as follows. In Section 2 we state existence and unicity theorem. In Section 3 we state averaging theorems. In Section 4 we study the corresponding Green functions. In section 5 we give the principal proofs. In Appendix we prove some technical results.

\section{Existence and unicity theorem}

In this section we study the strong solutions of the equation (1.7) with an arbitrary Brownian motion $\left(W_{t}\right)_{0 \leq t \leq 1}$. A stochastic process $\left(x_{t}\right)_{0 \leq t \leq 1}$ is called the random solution of the equation (1.7) if it satisfies this equation almost sure and for any $0 \leq t \leq 1$ the random variable $x_{t}$ is measurable with respect to $\sigma\left\{W_{u}, 0 \leq u \leq 1\right\}$. Indeed, the solution may be don't adapted with respect to the Wiener filtration.

The first problem is to find sufficient conditions for existence of unique strong solution. To this end we assume that the function $A(t, x)$ satisfies the following conditions.

$\mathbf{C}_{1}$ ) There exists a positive constant $p$ such that

$$
A(t, x)=p x+B(t, x),
$$

where the function $B(t, x)$ is bounded, i.e.

$$
\beta^{*}=\sup _{0 \leq t \leq 1} \sup _{x \in \mathbb{R}}|B(t, x)|<\infty .
$$

$\left.\mathbf{C}_{2}\right)$ The partial derivative $B_{x}^{\prime}(t, x)$ is bounded by $p$, i.e.

$$
\beta=\sup _{0 \leq t \leq 1} \sup _{x \in \mathbb{R}}\left|B_{x}^{\prime}(t, x)\right|<p
$$


and, moreover, it is uniformly continuous, i.e.

$$
\lim _{\delta \rightarrow 0} \sup _{0 \leq t \leq 1} \sup _{|x-y| \leq \delta}\left|B_{x}^{\prime}(t, x)-B_{x}^{\prime}(t, y)\right|=0 .
$$

For example, we can take $B(t, x)=\beta^{*} \sin (\omega t x)$ for $0 \leq \beta^{*}|\omega|<p$.

Theorem 2.1. Assume that the conditions $\left.\mathbf{C}_{1}\right)-\mathbf{C}_{2}$ ) hold. Then there exists nonrandom parameter $\varepsilon_{0}>0$ such that for all $0<\varepsilon \leq \varepsilon_{0}$ the equation (1.7) with one of the boundary conditions (1.2), (1.3) or (1.4) has unique continuously differentiable almost sure random solution.

The proof of this theorem is given in Section 5 .

Remark 2.1. It should be noted that the well-known example of a stochastic process satisfying a stochastic differential equation of a special type with the boundary conditions (1.2) is the Brownian Bridge (see, for example, [15]), which is defined as

$$
Z_{t}=W_{t}-t W_{1}=\int_{0}^{1} G(t, s) \mathrm{d} W_{s}
$$

where

$$
G(t, s)=(1-t) \mathbf{1}_{\{s \leq t\}}-t \mathbf{1}_{\{s>t\}} .
$$

Taking into account that $t^{-1}\left(Z_{t}-W_{t}\right)=-W_{1}$ for any $0 \leq t \leq 1$, we obtain

$$
\int_{0}^{t} s^{-1}\left(Z_{s}-W_{s}\right) \mathrm{d} s=-t W_{1}=Z_{t}-W_{t}
$$

i.e.

$$
\mathrm{d} Z_{t}=t^{-1}\left(Z_{t}-W_{t}\right) \mathrm{d} t+\mathrm{d} W_{t}, \quad Z_{0}=Z_{1}=0 .
$$

It is clear that the process (2.4) is not adapted with respect to the Wiener filtration. But it should be noted that for any one boundary condition there exist the backward stochastic differential equations with the adapted solutions, (see, for example, [9]). In this paper we investigate the second order differential equations of the form (1.7) with the two boundary conditions, whose solutions, generally speaking, can be not adapted with respect to the filtration generated by the Wiener process. 


\section{Averaging theorems}

In this section we study the asymptotic (as $\varepsilon \rightarrow 0$ ) properties of the solutions of the equation (1.7) with boundary conditions (1.2)-(1.4). To state the first theorem we set the following process

$$
\varkappa(t)=\int_{0}^{1} \Upsilon(t, s)(B(s, 0)+f(s)) \mathrm{d} s+\int_{0}^{1} \Upsilon(t, s) \delta(s) \mathrm{d} W_{s},
$$

where $\Upsilon(t, s)=-\min (t, s)(1-\max (t, s))$.

In the sequel we denote by $|\cdot|_{*}$ the uniform norm in $\mathbf{C}([0,1])$ and in $\mathbf{C}([0,1] \times[0,1])$, i.e. for any $x \in \mathbf{C}([0,1])$ and $G \in \mathbf{C}([0,1] \times[0,1])$

$$
|x|_{*}=\sup _{0 \leq t \leq 1}|x(t)| \text { and }|G|_{*}=\sup _{0 \leq t, s \leq 1}|G(t, s)| \text {. }
$$

Moreover, for any bounded $[0,1] \times[0,1] \rightarrow \mathbb{R}$ function $G$ we set

$$
|G|_{1, *}=\sup _{s \neq t, s, t \in[0,1]}|G(t, s)| .
$$

Theorem 3.1. Assume that the conditions $\left.\mathbf{C}_{1}\right)-\mathbf{C}_{2}$ ) hold. Then the random solution of the problem (1.7)-(1.2) possess the following limiting form

$$
\mathbf{P}-\lim _{\varepsilon \rightarrow 0}\left|\varepsilon^{-2} x^{\varepsilon}-\varkappa\right|_{*}=0 .
$$

Now we introduce the function

$$
B_{0}(x)=x+\frac{1}{p} \int_{0}^{1} B(s, x) \mathrm{d} s
$$

and we define the random variable (if it exists)

$$
\zeta=B_{0}^{-1}(\eta)
$$

where

$$
\eta=-\frac{1}{p} \int_{0}^{1} f(s) \mathrm{d} s-\frac{1}{p} \int_{0}^{1} \delta(s) \mathrm{d} W_{s} .
$$

Theorem 3.2. Assume that the conditions $\left.\mathbf{C}_{1}\right)-\mathbf{C}_{2}$ ) hold. Then the function (3.4) is invertible on $\mathbb{R}$. Moreover, the solutions of the problems (1.7)-(1.3) and (1.7)-(1.4) satisfy the following property

$$
\mathbf{P}-\lim _{\varepsilon \rightarrow 0}\left|x^{\varepsilon}-\zeta\right|_{*}=0 .
$$


Remark 3.1. It is easy to see, that for $\delta \equiv 0$ and $B(t, x) \equiv 0$ we obtain the convergence (1.5).

Remark 3.2. It should be noted that the theorems 2.1, 3.1-3.2 are true for any finite dimension state space also.

\section{Properties of the Green functions}

Let $p>0$ be arbitrary fixed constant. We make use of the following differential equation

$$
\ddot{u}-\varepsilon^{2} p u=0 .
$$

One can check directly, that for the problem (4.1) - (1.2) the Green function is defined as

$$
G_{1, \varepsilon}(t, s)=-\frac{e^{-\varepsilon \sqrt{p}|t-s|} g_{1, \varepsilon}(t, s) g_{2, \varepsilon}(t, s)}{2 \varepsilon \sqrt{p}\left(1-e^{-2 \varepsilon \sqrt{p}}\right)}
$$

where

$$
g_{1, \varepsilon}(t, s)=1-e^{-2 \varepsilon \sqrt{p} \min (t, s)} \quad \text { and } \quad g_{2, \varepsilon}(t, s)=1-e^{-2 \varepsilon \sqrt{p}(1-\max (t, s))} .
$$

Proposition 4.1. The function (4.2) satisfies the following limit properties

$$
\lim _{\varepsilon \rightarrow 0}\left|G_{1, \varepsilon}-\Upsilon\right|_{*}=0
$$

and

$$
\lim _{\varepsilon \rightarrow 0}\left|\frac{\partial}{\partial t}\left(G_{1, \varepsilon}-\Upsilon\right)\right|_{1, *}=0
$$

where the norms $|\cdot|_{*}$ and $|\cdot|_{1, *}$ are defined in (3.2) and (3.3).

For the problem (4.1) - (1.3) the Green function is

$$
G_{2, \varepsilon}(t, s)=-\frac{e^{-\varepsilon \sqrt{p}|t-s|} g_{3, \varepsilon}(t, s) g_{4, \varepsilon}(t, s)}{2 \varepsilon \sqrt{p}\left(1-e^{-2 \varepsilon \sqrt{p}}\right)},
$$

where

$$
g_{3, \varepsilon}(t, s)=1+e^{-2 \varepsilon \sqrt{p} \min (t, s)} \quad \text { and } \quad g_{4, \varepsilon}(t, s)=1+e^{-2 \varepsilon \sqrt{p}(\max (t, s))} .
$$


Moreover, for the problem (4.1) - (1.4) the Green function has the form

$$
G_{3, \varepsilon}(t, s)=-\frac{e^{-\varepsilon \sqrt{p}|t-s|}+e^{-\varepsilon \sqrt{p}(1-|t-s|)}}{2 \varepsilon \sqrt{p}\left(1-e^{-\varepsilon \sqrt{p}}\right)} .
$$

Proposition 4.2. The functions (4.5) and (4.6) satisfy the following limit properties

$$
\lim _{\varepsilon \rightarrow 0} \max _{2 \leq i \leq 3}\left|\varepsilon^{2} G_{i, \varepsilon}+\frac{1}{p}\right|_{*}=0
$$

and

$$
\lim _{\varepsilon \rightarrow 0} \varepsilon^{2} \max _{2 \leq i \leq 3}\left|\frac{\partial}{\partial t} G_{i, \varepsilon}\right|_{1, *}=0
$$

\section{Proofs}

\subsection{Proof of Theorem 2.1}

We define for $1 \leq i \leq 3$ the following $\mathcal{C}[0,1] \rightarrow \mathcal{C}[0,1]$ operators

$$
\Psi_{i}(\varepsilon, x)(t)=x(t)-\int_{0}^{1} \widetilde{G}_{i, \varepsilon}(t, s) B(s, x(s)) \mathrm{d} s,
$$

where $\widetilde{G}_{i, \varepsilon}(t, s)=\varepsilon^{2} G_{i, \varepsilon}(t, s)$ and the corresponding Green functions $G_{i, \varepsilon}$ are defined in (4.2), (4.5) and (4.6) respectively and the function $B(t, x)$ is given in the condition $\mathbf{C}_{1}$ ).

To show this theorem we have to check the conditions of Theorem A.3. Note that

$$
\Psi_{1}(0, x)(t)=x(t)
$$

and

$$
\Psi_{2}(0, x)(t)=\Psi_{3}(0, x)(t)=x(t)+\frac{1}{p} \int_{0}^{1} B(s, x(s)) \mathrm{d} s .
$$

Therefore, the conditions $\left.\mathbf{A} \mathbf{P}_{1}\right)-\mathbf{A P}_{2}$ ) are obvious for $i=1$. Let us check these conditions for $i=2$ and $i=3$. To this end we introduce for any function $\phi \in \mathcal{C}[0,1]$ the $\mathbb{R} \rightarrow \mathbb{R}$ function

$$
T_{\phi}(v)=-\frac{1}{p} \int_{0}^{1} B(s, \phi(s)+v) \mathrm{d} s .
$$


It is clear that

$$
T_{\phi}^{\prime}(v)=-\frac{1}{p} \int_{0}^{1} B_{x}^{\prime}(s, \phi(s)+v) \mathrm{d} s
$$

and by the inequality $(2.2)$

$$
\sup _{\phi \in \mathcal{C}[0,1]} \sup _{v \in \mathbb{R}}\left|T_{\phi}^{\prime}(v)\right|<1
$$

Therefore, for any $\phi \in \mathcal{C}[0,1]$ the equation

$$
v=T_{\phi}(v)
$$

has an unique solution. This implies directly that in tis case for any $\phi \in$ $\mathcal{C}[0,1]$ the inverse function in the condition $\left.\mathbf{A P}_{1}\right)$ is given as

$$
x_{0}^{\phi}(t)=\phi(t)+v_{\phi}
$$

where $v_{\phi}$ is the solution of the equation (5.3).

As to the condition $\mathbf{A P}_{2}$ ), note that, the Fréchet derivative of the function $(5.2)$ is given by the following linear $\mathcal{C}[0,1] \rightarrow \mathcal{C}[0,1]$ operator

$$
D_{x}(h)(t)=h(t)+\frac{1}{p} \int_{0}^{1} B_{x}^{\prime}(s, x(s)) h(s) \mathrm{d} s .
$$

Taking into account the inequality (2.1) we can directly check that this operator is isomorphism and

$$
D_{x}^{-1}(h)(t)=h(t)-\frac{\int_{0}^{1} B_{x}^{\prime}(s, x(s)) h(s) \mathrm{d} s}{p+\int_{0}^{1} B_{x}^{\prime}(s, x(s)) \mathrm{d} s} .
$$

Now we calculate directly, that for any $x \in \mathbf{C}[0,1]$

$$
\left\|D_{x}^{-1}\right\|=\sup _{h \in \mathbf{C}[0,1],|h|_{*}=1}\left|D_{x}^{-1}(h)\right| \leq 1+\frac{\beta}{p-\beta} .
$$

This implies condition $\mathbf{A P}_{2}$ ).

Moreover, the boundedness of the function $B(t, x)$ given in the condition $\mathbf{C}_{1}$ ) implies the condition $\mathbf{A P}_{3}$ ) for all $1 \leq i \leq 3$. Condition $\mathbf{C}_{2}$ ) enables the 
conditions $\mathbf{A P}_{4}$ ) and $\mathbf{A} \mathbf{P}_{5}$ ). Therefore, by making use of Theorem A.3 for the random functions

$$
\phi_{i, \varepsilon}(t)=\int_{0}^{1} \widetilde{G}_{i, \varepsilon}(t, s) f(s) \mathrm{d} s+\int_{0}^{1} \widetilde{G}_{i, \varepsilon}(t, s) \delta(s) \mathrm{d} W_{s}
$$

we obtain that there exists some nonrandom parameter $\varepsilon_{*}>0$ such that for any $0 \leq \varepsilon \leq \varepsilon_{*}$ and for any $1 \leq i \leq 3$ there exist random functions $x_{i}^{\varepsilon} \in \mathcal{C}[0,1]$ for which

$$
\Psi_{i, \varepsilon}\left(\varepsilon, x_{i}^{\varepsilon}\right)=\phi_{i, \varepsilon}
$$

i.e.

$$
\begin{aligned}
x_{i}^{\varepsilon}(t) & =\int_{0}^{1} \widetilde{G}_{i, \varepsilon}(t, s) B\left(s, x_{i}^{\varepsilon}(s)\right) \mathrm{d} s+\int_{0}^{1} \widetilde{G}_{i, \varepsilon}(t, s) f(s) \mathrm{d} s \\
& +\int_{0}^{1} \widetilde{G}_{i, \varepsilon}(t, s) \delta(s) \mathrm{d} W_{s} .
\end{aligned}
$$

By Lemma A.2 this function satisfies the stochastic differential equation (1.7) with $i$ th boundary condition in (1.2)-(1.4).

Now we show that this solution is unique. To this end note that through Lemma A.2 we obtain that the uniqueness of the equation (1.7) is equivalent to the uniqueness of the equation (5.4). Moreover, taking into account the asymptotic properties (4.3), (4.7) and the inequality (2.2) we can find some parameters $0<\varepsilon_{0} \leq \varepsilon_{*}$ and $0<\theta<1$ such that

$$
\max _{1 \leq i \leq 3} \sup _{0<\varepsilon \leq \varepsilon_{0}} \sup _{0 \leq t, s \leq 1} \sup _{x, y \in \mathbb{R}}\left|\widetilde{G}_{i, \varepsilon}(t, s)\right| \frac{|B(s, x)-B(s, y)|}{|x-y|} \leq \theta .
$$

This implies immediately that the equation (5.4) has unique solution. Hence Theorem 2.1.

\subsection{Proof of Theorem 3.1}

First, we set

$$
\bar{x}^{\varepsilon}(t)=\frac{1}{\varepsilon^{2}} x_{1}^{\varepsilon}(t)-\varkappa(t) \quad \text { and } \quad \bar{G}_{\varepsilon}(t, s)=G_{1, \varepsilon}(t, s)-\Upsilon(t, s) .
$$


By making use of the representation (5.4) for $i=1$, we obtain

$$
\bar{x}^{\varepsilon}(t)=\int_{0}^{1}\left(G_{1, \varepsilon}(t, s) B\left(s, x_{1}^{\varepsilon}(s)\right)-\Upsilon(t, s) B(s, 0)\right) \mathrm{d} s+\varsigma_{t}^{\varepsilon},
$$

where $\varsigma_{t}^{\varepsilon}=\int_{0}^{1} \bar{G}_{1, \varepsilon}(t, s) f(s) \mathrm{d} s+\eta_{t}^{\varepsilon}$ and

$$
\eta_{t}^{\varepsilon}=\int_{0}^{1} \bar{G}_{\varepsilon}(t, s) \delta(s) \mathrm{d} W_{s} .
$$

It is clear that the property (4.3) yields

$$
\lim _{\varepsilon \rightarrow 0} \sup _{0 \leq t \leq 1} \int_{0}^{1} \bar{G}_{1, \varepsilon}(t, s) f(s) \mathrm{d} s=0 .
$$

Furthermore, taking into account that

$$
\Delta \bar{G}_{\varepsilon}(t, t)=\Delta \bar{G}_{\varepsilon}(t, t)-\Delta \bar{G}_{\varepsilon}(t-0, t)=0,
$$

Lemma A.1 yields

$$
\eta_{t}^{\varepsilon}=\eta_{0}^{\varepsilon}+\int_{0}^{t} \bar{D}_{\varepsilon}(s) \mathrm{d} s
$$

where

$$
\bar{D}_{\varepsilon}(s)=\int_{0}^{1} \bar{G}_{1, \varepsilon}(t, s) \delta(s) \mathrm{d} W_{s} \quad \text { and } \quad \bar{G}_{1, \varepsilon}(t, s)=\frac{\partial}{\partial t} \bar{G}_{\varepsilon}(t, s) .
$$

First of all, note that in view of the property (4.3)

$$
\mathbf{E}\left(\eta_{0}^{\varepsilon}\right)^{2}=\int_{0}^{1} \bar{G}_{\varepsilon}^{2}(0, s) \delta^{2}(s) \mathrm{d} s \leq\left|\bar{G}_{\varepsilon}\right|_{*}^{2}\|\delta\|^{2} \rightarrow 0
$$

as $\varepsilon \rightarrow 0$. Moreover,

$$
\sup _{0 \leq s \leq 1} \mathbf{E}\left(\bar{D}_{\varepsilon}(s)\right)^{2} \leq\left|\bar{G}_{1, \varepsilon}\right|_{1, *}^{2}
$$

and, therefore, in view of the property (4.4)

$$
\lim _{\varepsilon \rightarrow 0} \sup _{0 \leq s \leq 1} \mathbf{E}\left(\bar{D}_{\varepsilon}(s)\right)^{2}=0
$$


Thus,

$$
\mathbf{P}-\lim _{\varepsilon \rightarrow 0}\left|\eta^{\varepsilon}\right|_{*}=0 \quad \text { and } \quad \mathbf{P}-\lim _{\varepsilon \rightarrow 0}\left|\varsigma^{\varepsilon}\right|_{*}=0 .
$$

Now we rewrite the equality (5.5) as

$$
\begin{aligned}
\bar{x}^{\varepsilon}(t) & =\int_{0}^{1} \Upsilon(t, s)\left(B\left(s, x_{1}^{\varepsilon}(s)\right)-B(s, 0)\right) \mathrm{d} s \\
& +\int_{0}^{1} \bar{G}_{\varepsilon}(t, s) B\left(s, x_{1}^{\varepsilon}(s)\right) \mathrm{d} s+\varsigma_{t}^{\varepsilon} .
\end{aligned}
$$

Taking into account here the conditions $\mathbf{C}_{1}$ ) and $\mathbf{C}_{2}$ ) we obtain that

$$
\begin{aligned}
\left|\bar{x}^{\varepsilon}\right|_{*} & \leq \beta|\Upsilon|_{*}\left|x_{1}^{\varepsilon}\right|_{*}+\beta^{*}\left|\overline{G_{\varepsilon}}\right|_{*}+\left|\varsigma^{\varepsilon}\right|_{*} \\
& \leq \varepsilon^{2} \beta|\Upsilon|_{*}\left|\bar{x}^{\varepsilon}\right|_{*}+\varepsilon^{2} \beta|\Upsilon|_{*}|\varkappa|_{*}+\beta^{*}\left|\bar{G}_{\varepsilon}\right|_{*}+\left|\varsigma^{\varepsilon}\right|_{*} .
\end{aligned}
$$

Therefore, for sufficiently small $\varepsilon$ we get

$$
\left|\bar{x}^{\varepsilon}\right|_{*} \leq \frac{1}{1-\varepsilon^{2} \beta|\Upsilon|_{*}}\left(\varepsilon^{2} \beta|\Upsilon|_{*}|\varkappa|_{*}+\beta^{*}\left|\bar{G}_{\varepsilon}\right|_{*}+\left|\varsigma^{\varepsilon}\right|_{*}\right) \rightarrow 0
$$

as $\varepsilon \rightarrow 0$. Hence Theorem 3.1.

\subsection{Proof of Theorem 3.2}

First of all note that the boundedness of the function $B(s, x)$ in the condition $\mathbf{C}_{1}$ ) implies

$$
\lim _{x \rightarrow+\infty} B_{0}(x)=+\infty \quad \text { and } \quad \lim _{x \rightarrow-\infty} B_{0}(x)=-\infty
$$

Moreover, by the inequality (2.2) we get

$$
\mathbf{b}_{*}=\inf _{x \in \mathbb{R}}\left|B_{0}^{\prime}(x)\right|>0 .
$$

These properties imply that for any $a \in \mathbb{R}$ the equation

$$
B_{0}(x)=a
$$

has an unique solution, i.e. the function $B_{0}$ defined in (3.4) is invertible on $\mathbb{R}$. Moreover, the representation (5.4) implies

$$
B_{0}\left(x^{\varepsilon}(t)\right)=\eta+u^{\varepsilon}(t)+v^{\varepsilon}(t),
$$


where

$$
u^{\varepsilon}(t)=\int_{0}^{1} V^{\varepsilon}(t, s)\left(B\left(s, x^{\varepsilon}(s)\right)+f(s)\right) \mathrm{d} s
$$

and

$$
v^{\varepsilon}(t)=\int_{0}^{1} V^{\varepsilon}(t, s) \delta(s) \mathrm{d} W_{s} \quad \text { with } \quad V^{\varepsilon}(t, s)=\widetilde{G}_{\varepsilon}(t, s)+\frac{1}{p} .
$$

The limit (4.7) implies directly

$$
\mathbf{P}-\lim _{\varepsilon \rightarrow 0} \sup _{0 \leq t \leq 1}\left|u^{\varepsilon}(t)\right|=0 .
$$

Now we show that

$$
\mathbf{P}-\lim _{\varepsilon \rightarrow 0} \sup _{0 \leq t \leq 1}\left|v^{\varepsilon}(t)\right|=0 .
$$

Indeed, by Lemma A.1 process $v^{\varepsilon}(t)$ is almost sure continuously differentiable and taking into account that $\Delta V^{\varepsilon}(t, t)=0$ we find that

$$
v^{\varepsilon}(t)=v^{\varepsilon}(0)+\int_{0}^{t} \check{D}_{\varepsilon}(s) \mathrm{d} s
$$

where

$$
\check{D}_{\varepsilon}(s)=\int_{0}^{1} \frac{\partial}{\partial s} V^{\varepsilon}(s, \vartheta) \delta(\vartheta) \mathrm{d} W_{\vartheta}=\int_{0}^{1} \frac{\partial}{\partial s} \widetilde{G}_{\varepsilon}(s, \vartheta) \delta(\vartheta) \mathrm{d} W_{\vartheta} .
$$

Recall, that $\widetilde{G}_{\varepsilon}$ is equal to $\widetilde{G}_{2, \varepsilon}$ or $\widetilde{G}_{3, \varepsilon}$. So, by (4.7)

$$
\mathbf{E}\left(v^{\varepsilon}(0)\right)^{2}=\int_{0}^{1}\left(V^{\varepsilon}(0, s)\right)^{2} \mathrm{~d} s \rightarrow 0 \quad \text { as } \quad \varepsilon \rightarrow 0
$$

and by (4.8)

$$
\sup _{0 \leq s \leq 1} \mathbf{E}\left(\check{D}_{\varepsilon}(s)\right)^{2}=\sup _{0 \leq s \leq 1} \int_{0}^{1}\left(\frac{\partial}{\partial s} \widetilde{G}_{\varepsilon}(s, \vartheta)\right)^{2} \mathrm{~d} \vartheta \rightarrow 0 \quad \text { as } \quad \varepsilon \rightarrow 0 .
$$

This implies immediately (5.10). Therefore, taking into account that

$$
B_{0}(\zeta)=\eta
$$

and that

$$
|x-y| \leq \frac{1}{\mathbf{b}_{*}}\left|B_{0}(x)-B_{0}(y)\right|
$$

we obtain Theorem 3.2. 


\section{Conclusion}

In this paper we introduced and studied the second oder differential equations containing brownian motion and a small parameter with boundary conditions. We proved an existence and unicity theorem for random solutions and we shown an average principle as the small parameter goes to zero. Through the Green functions method we obtained the explicit limit forms for solutions.

Acknowledgements. The first author is partially supported by the Ministry of Education and Science of the Russian Federation in the framework of the research project No 1.3464.2017 and by RFBR Grant 16-01-00386. The second author is partially supported by the RSF grant 14-49-00079 (National Research University "MPEI" 14 Krasnokazarmennaya, 111250 Moscow, Russia), by RFBR Grant 16-01-00121, by the Ministry of Education and Science of the Russian Federation in the framework of the research project No 2.3208.2017/PCH, by the Russian Federal Professor program (Project No 1.472.2016/FPM, Ministry of Education and Science) and by the Academic D.I. Mendeleev Fund Program of the Tomsk State University (research project NU 8.1.55.2015 L).

\section{Appendix}

\section{A.1 Technical Lemmas}

Lemma A.1. Let $G(t, s)$ be a function having the following form

$$
G(t, s)= \begin{cases}\sum_{j=1}^{m} g_{1 j}(t) h_{1 j}(s) & \text { if } t \geq s ; \\ \sum_{j=1}^{m} g_{2 j}(t) h_{2 j}(s) & \text { if } t<s .\end{cases}
$$

Assume that the functions $g_{1 j}$ and $g_{2 j}$ belong to $\mathbf{C}^{2}[0,1]$. Then the stochastic process

$$
\xi_{t}=\int_{0}^{1} G(t, s) \delta(s) \mathrm{d} W_{s}
$$

admits the following Ito differential

$$
\mathrm{d} \xi_{t}=D(t) \mathrm{d} t+\Delta G(t, t) \delta(t) \mathrm{d} W_{t},
$$


where

$$
D(t)=\int_{0}^{1} G_{1}(t, s) \delta(s) \mathrm{d} W_{s} \quad \text { with } \quad G_{1}(t, s)=\frac{\partial}{\partial t} G(t, s)
$$

and

$$
\Delta G(t, t)=G(t, t)-G(t-0, t)=\sum_{j=1}^{m} g_{1 j}(t) h_{1 j}(t)-\sum_{j=1}^{m} g_{2 j}(t) h_{2 j}(t) .
$$

Proof. First, note that

$$
\xi_{t}=\int_{0}^{t} G(t, s) \delta(s) \mathrm{d} W_{s}+\int_{t}^{1} G(t, s) \delta(s) \mathrm{d} W_{s} .
$$

Taking into account here the definition of the function $G$ we get

$$
\begin{aligned}
\xi_{t} & =\sum_{j=1}^{m} g_{1 j}(t) \int_{0}^{t} h_{1 j}(s) \mathrm{d} W_{s}+\sum_{j=1}^{m} g_{2 j}(t) \int_{t}^{1} h_{2 j}(s) \mathrm{d} W_{s} \\
& =\sum_{j=1}^{m} g_{1 j}(t) \int_{0}^{t} h_{1 j}(s) \mathrm{d} W_{s}+\sum_{j=1}^{m} g_{2 j}(t) \int_{0}^{1} h_{2 j}(s) \mathrm{d} W_{s} \\
& -\sum_{j=1}^{m} g_{2 j}(t) \int_{0}^{t} h_{2 j}(s) \mathrm{d} W_{s} .
\end{aligned}
$$

Now, this Lemma follows directly from the Ito formula.

Now we make use of the following conditions for the Green functions $\left.\mathbf{H}_{1}\right)$ The $[0,1] \times[0,1] \rightarrow \mathbb{R}$ function $G(\cdot, \cdot)$ is continuous and has the following form

$$
G(t, s)= \begin{cases}g_{11}(t) h_{11}(s)+g_{12}(t) h_{12}(s) & \text { if } t \geq s \\ g_{21}(t) h_{21}(s)+g_{22}(t) h_{22}(s) & \text { if } t<s\end{cases}
$$

where the functions $g_{11}(\cdot), g_{12}(\cdot), g_{21}(\cdot) g_{22}(\cdot)$ are two times continuously differentiable such that

$$
\frac{\partial^{2}}{\partial t^{2}} G(t, s)-p(t) G(t, s)=0
$$

for any $t \neq s$ from $[0,1]$. 
$\mathbf{H}_{2}$ ) The first partial derivative of the function $G$ has the jump

$$
\frac{\partial}{\partial t} G(t, t)-\frac{\partial}{\partial t} G(t-0, t)=1 .
$$

Lemma A.2. Assume that the function $G$ satisfies the conditions $\left.\mathbf{H}_{1}\right)-\mathbf{H}_{2}$ ) and $\varphi$ is arbitrary function from $\mathbf{C}[0,1]$. Then, almost sure, the stochastic process

$$
\xi_{t}=\int_{0}^{1} G(t, s) \varphi(s) \mathrm{d} s+\int_{0}^{1} G(t, s) \delta(s) \mathrm{d} W_{s}
$$

has continuous derivative and satisfies the following second order stochastic equation

$$
\ddot{\xi}_{t}=p(t) \xi_{t}+\varphi(t)+\delta(t) \dot{W}_{t},
$$

i.e. the derivative $\dot{\xi}_{t}$ satisfies the following stochastic differential equation

$$
\mathrm{d} \dot{\xi}_{t}=p(t) \xi_{t} \mathrm{~d} t+\varphi(t) \mathrm{d} t+\delta(t) \mathrm{d} W_{t} .
$$

Proof. First of all we set

$$
\mathbf{a}_{t}=\int_{0}^{1} G(t, s) \varphi(s) \mathrm{d} s \quad \text { and } \quad \mathbf{b}_{t}=\int_{0}^{1} G(t, s) \delta(s) \mathrm{d} W_{s} .
$$

It is easy to check directly that

$$
\ddot{\mathbf{a}}_{t}=p(t) \mathbf{a}_{t}+\varphi(t) .
$$

Moreover, note that Condition $\mathbf{H}_{1}$ ) implies $\Delta G(t, t)=0$. Therefore, by Lemma A.1 we obtain

$$
\mathbf{b}_{t}=\int_{0}^{t} D(s) \mathrm{d} s
$$

where the process $D(t)$ is defined in (A.1). From here we obtain that

$$
\dot{\mathbf{b}}_{t}=D(t)=\int_{0}^{1} G_{1}(t, s) \delta(s) \mathrm{d} W_{s} .
$$

Therefore, by Lemma A.1 we find

$$
\mathrm{d} \dot{\mathbf{b}}_{t}=D_{1}(t) \mathrm{d} t+\Delta G_{1}(t, t) \delta(t) \mathrm{d} W_{t},
$$


where

$$
D_{1}(t)=\int_{0}^{1} \frac{\partial}{\partial t} G_{1}(t, s) \delta(s) \mathrm{d} W_{s}=\int_{0}^{1} \frac{\partial^{2}}{\partial t^{2}} G(t, s) \delta(s) \mathrm{d} W_{s} .
$$

Now in view of the equations (A.3) and the definition (A.5) we obtain that

$$
D_{1}(t)=p(t) \mathbf{b}(t) \text {. }
$$

Therefore, the condition $\mathbf{H}_{2}$ ) implies this lemma.

\section{A.2 Green functions}

In this section we consider the second order $\mathbf{C}^{2}[0,1] \rightarrow \mathbf{C}^{2}[0,1]$ linear differential operator $L$ defines as

$$
L(x)(t)=\ddot{x}(t)+p(t) \dot{x}(t)+q(t) x(t)
$$

with the boundary conditions

$$
\alpha_{1} x(0)+\beta_{1} \dot{x}(0)=0 \quad \text { and } \quad \alpha_{2} x(1)+\beta_{2} \dot{x}(1)=0,
$$

where $\alpha_{1}, \beta_{1}, \alpha_{2}$ and $\beta_{2}$ are some fixed constants. We will consider also the following mixed conditions

$$
x(0)=x(1) \quad \text { and } \quad \dot{x}(0)=\dot{x}(1) .
$$

The operator $L$ is called regular if the equation

$$
L(x)=0
$$

with the boundary conditions (A.6) has only trivial solution $x \equiv 0$. If the operator $L$ is regular, then the problem

$$
L(x)=f
$$

with the boundary conditions (A.6) may be written as

$$
x(t)=\int_{0}^{1} G(t, s) f(s) \mathrm{d} s
$$

(see, for example, [19], p. 39). Here the $[0,1]^{2} \rightarrow \mathbb{R}$ function $G$ is called Green function generated by the operator $L$ and the boundary conditions (A.6). The function $G$ has the following properties 
1. $G(t, s)$ is continuous with respect to $t$ and $s$.

2. For $t \neq s$ the function $G(t, s)$ is two times continuously differentiable with respect to $t$ and $s$. Moreover, for $t \neq s$

$$
\frac{\partial^{2}}{\partial t^{2}} G(t, s)+p(t) \frac{\partial}{\partial t} G(t, s)+q(t) G(t, s)=0
$$

and

$$
\alpha_{1} G(0, s)+\beta_{1} \frac{\partial}{\partial t} G(0, s)=0 \quad \text { and } \quad \alpha_{1} G(1, s)+\beta_{1} \frac{\partial}{\partial t} G(1, s)=0,
$$

or

$$
G(0, s)=G(1, s) \quad \text { and } \quad \frac{\partial}{\partial t} G(0, s)=\frac{\partial}{\partial t} G(1, s)
$$

3. The function $G$ is symmetric, i.e.

$$
G(t, s)=G(s, t)
$$

4. The partial derivative with respect to $t$ has the unitary jump, i.e.

$$
\frac{\partial}{\partial t} G(t+0, t)-\frac{\partial}{\partial t} G(t-0, t)=1 .
$$

\section{A.3 Uniform implicit function theorem}

Let $F$ be a Banach space and $\Psi:[0,1] \times F \rightarrow F$ be a continuous function. In this section we study the following equation

$$
\Psi(\varepsilon, x)=\phi \quad \text { for } \quad \phi \in F .
$$

$\mathbf{A P}_{1}$ ) The equation (A.9) for $\varepsilon=0$ has unique solution $x_{0}^{\phi}$ for any $\phi \in F$. $\left.\mathbf{A P}_{2}\right)$ For any $\phi \in F$ the partial Fréchet derivative

$$
\Upsilon(\phi)=\frac{\partial}{\partial x} \Psi\left(0, x_{0}^{\phi}\right)
$$

is $F \rightarrow F$ isomorphism and

$$
\sup _{\phi \in F}\left|\Upsilon^{-1}(\phi)\right|<\infty
$$


$\mathbf{A P}_{3}$ ) The function $\Psi$ is uniform continuous in $\varepsilon$, i.e.

$$
\lim _{\varepsilon \rightarrow 0} \sup _{x \in F}|\Psi(\varepsilon, x)-\Psi(0, x)|=0 .
$$

$\left.\mathbf{A P}_{4}\right)$ The function $\Psi$ is uniformly differentiable in the Fréchet sense with respect to $x$, i.e.

$$
\lim _{\delta \rightarrow 0} \sup _{0<\varepsilon \leq 1} \sup _{|x-y| \leq \delta} \frac{\left|\Psi(\varepsilon, y)-\Psi(\varepsilon, x)-\frac{\partial}{\partial x} \Psi(\varepsilon, x)(y-x)\right|}{|x-y|}=0 .
$$

$\left.\mathbf{A P}_{5}\right)$ The partial Fréchet derivative of $\partial \Psi / \partial x$ is uniform continuous, i.e.

$$
\lim _{\varepsilon \rightarrow 0} \limsup _{\delta \rightarrow 0} \sup _{|x-y|<\delta}\left|\frac{\partial}{\partial x} \Psi(\varepsilon, x)-\frac{\partial}{\partial x} \Psi(0, y)\right|=0 .
$$

Theorem A.3. Assume that the conditions $\left.\left.\mathbf{A P}_{1}\right)-\mathbf{A P}_{5}\right)$ hold. Then there exist $\varepsilon_{0} \in(0,1)$ and $\delta>0$ such that for any $0<\varepsilon \leq \varepsilon_{0}$ and any $\phi \in F$ there exists unique $x_{\varepsilon}^{\phi} \in \mathbf{B}\left(x_{0}^{\phi}, \delta\right)$ which satisfies the equation (A.9), i.e.

$$
\Psi\left(\varepsilon, x_{\varepsilon}^{\phi}\right)=\phi
$$

where $\mathbf{B}(a, \delta)=\{x \in F:|x-a| \leq \delta\}$.

Proof. By the same way as in [8](page 57) we introduce the following special function

$$
F(\varepsilon, z)=z-\Upsilon^{-1}(\phi)(\Psi(\varepsilon, z)-\phi)
$$

Note that the conditions $\left.\left.\mathbf{A} \mathbf{P}_{2}\right), \mathbf{A} \mathbf{P}_{4}\right)$ and $\left.\mathbf{H}_{5}\right)$ imply that there exist $\varepsilon_{*}>0$, $\delta>0$ and $0<\theta<1$ such that for any $0 \leq \varepsilon \leq \varepsilon_{0}$ and $x, y$ from $\mathbf{B}\left(x_{0}^{\phi}, \delta\right)$

$$
|F(\varepsilon, x)-F(\varepsilon, y)| \leq \theta|x-y|
$$

Now we define the following approximating sequence

$$
z_{n}^{\varepsilon}=F\left(\varepsilon, z_{n-1}^{\varepsilon}\right) \quad \text { for } \quad n \geq 1
$$

and $z_{0}^{\varepsilon}=x_{0}^{\phi}$. First of all note that

$$
\left|z_{1}-x_{0}^{\phi}\right|=\left|z_{1}-z_{0}\right|=\left|\Upsilon^{-1}(\phi)\left(\Psi\left(\varepsilon, x_{0}^{\phi}\right)-\Psi\left(0, x_{0}^{\phi}\right)\right)\right|
$$


and by the inequality (A.10) and the condition $\mathbf{A P}_{3}$ ) we can choose $0<\varepsilon_{0} \leq$ $\varepsilon_{*}$ such that for all $0<\varepsilon \leq \varepsilon_{0}$

$$
\left|z_{1}^{\varepsilon}-z_{0}^{\varepsilon}\right| \leq(1-\theta) \delta<\delta,
$$

i.e. $z_{1}^{\varepsilon} \in \mathbf{B}\left(z_{0}, \delta\right)$. Now through the induction method we assume that $z_{j}^{\varepsilon} \in \mathbf{B}\left(z_{0}, \delta\right)$ for all $1 \leq j \leq n-1$ with $n \geq 2$. Let us check that in this case $z_{n}^{\varepsilon} \in \mathbf{B}\left(z_{0}, \delta\right)$ as well. Indeed, taking into account our condition implies directly the following inequality

$$
\left|z_{n}^{\varepsilon}-z_{n-1}^{\varepsilon}\right| \leq \theta\left|z_{n-1}^{\varepsilon}-z_{n-2}^{\varepsilon}\right| \leq \theta^{n-1}\left|z_{1}^{\varepsilon}-z_{0}^{\varepsilon}\right|
$$

we get

$$
\left|z_{n}^{\varepsilon}-x_{0}^{\phi}\right|=\left|z_{n}^{\varepsilon}-z_{0}^{\varepsilon}\right| \leq \sum_{j=1}^{n}\left|z_{j}^{\varepsilon}-z_{j-1}^{\varepsilon}\right| \leq \sum_{j=1}^{n} \theta^{j-1}\left|z_{1}^{\varepsilon}-z_{0}^{\varepsilon}\right| \leq \delta .
$$

Thus $z_{n}^{\varepsilon} \in \mathbf{B}\left(z_{0}, \delta\right)$ for all $n \geq 2$. Therefore, for any $n \geq 1$

$$
\left|z_{n}^{\varepsilon}-z_{n-1}^{\varepsilon}\right| \leq \theta\left|z_{n-1}^{\varepsilon}-z_{n-2}^{\varepsilon}\right|,
$$

i.e. for any $0 \leq \varepsilon \leq \varepsilon_{0}$ there exists the limit

$$
\lim _{n \rightarrow \infty} z_{n}^{\varepsilon}=z_{*}^{\varepsilon},
$$

which is the unique solution of the equation $z=F(\varepsilon, z)$ in the ball $\mathbf{B}\left(z_{0}, \delta\right)$ for $\varepsilon \leq \varepsilon_{0}$. This implies immediately Theorem A.3.

\section{References}

[1] Alvarez, O. and Bardi, M. (2001/02) Viscosity solutions methods for singular perturbations in deterministic and stochastic control. SIAM J. Control Optim. 40 (4), 11591188 
[2] Artzein, Z. and Gaitsgory, V. (2000) The value function of singularly perturbed control systems. Applied Mathematics and Optimization, $41,425-445$.

[3] Bensoussan, A. Perturbation Methods in Optimal Control. J. Wiley/Gauthier Villars, New York, 1988.

[4] Butuzov, V. F., Vasileva, A. B. and Fedoryuk, M. V. (1970) Asymptotic methods in the theory of ordinary differential equations. Progress in Mathematics, Mathematical Analysis, 8, 1-82, New York

[5] Ait-Sahalia, Y., Mykland, P.A. and Zhang, L. (2005) How often to sample a continuous-time process in the presence of market microstructure noise. Rev. Financ. Studies, 18, 351-416.

[6] Ait-Sahalia, Y., Mykland, P.A. and Zhang, L. (2005) A tale of two times scales: Determining integrated volatility with noisy highfrequency data. J. Amer. Statist. Assoc., 100, 1394-1411.

[7] Bogolubov, N.N. and Mitropol'skii, Yu.A. Asymptotic methods in the theory of nonlinear oscillation. M.: Fizmatgiz, 1963 (in Russian).

[8] Cartan, H. Cours de calcul différentiel. Hermann, Éditeurs des sciences et des arts, 1990.

[9] El Karoui, N. and Mazliak, L. Backward Stochastic differential equations. Pitman Research Notes in Mathematics Series, 364. Longman, Harlow, 1997.

[10] Fedoryuk, M.V. Asymptotic analysis: linear ordinary differential equations. Springer, Berlin, 1993.

[11] Fouque, J-P., Papanicolaou, G. and Sicar, K. Derivatives in financial markets with stochastic volatility. Cambridge University Press, 2000.

[12] Freidlin, M.I. and Wentzell, A.D. Random Perturbations of Dynamical Systems. Springer-Verlag, New York, 1986.

[13] Kabanov, Yu. and Pergamenshchikov, S. Two Scale Stochastic Systems: Asymptotic Analysis and Control. Applications of Mathematics, Stochastic Modeling and Applied Probability, 49, Springer-Verlag, Berlin, New York, 2003. 
[14] Kamenskii, M., Nistri, P. and Quincampoix, M. (2002) Sliding mode control of uncertain systems: a singular perturbation approach. IMA J. Math. Control Inform., 19 (4), 377-398.

[15] Karatzas, I. and Shreve, S.E. Brownian Motion and Stochastic Calculus. Springer-Verlag, 1991.

[16] Khasminskii, R. and Krylov, N. (2001) On averaging principle for diffusion processes with nullrecurrent fast component. Stoch Proc Appl. 93, 229-240.

[17] Kushner, H. Weak Convergence Methods and Singularly Perturbed Stochastic Control and Filtering. IEEE Press, New York, 1986.

[18] Lamberton, D. and Lapeyre, B. Introduction to stochastic calculus applied to finance. Chapman \& Hall, London, 1996.

[19] Naimark, M.A. Linear differential operators. (in Russian) / translated by E.R. Dawson ; English translation edited by W.N. Everitt, London; Toronto : Harrap, 1968.

[20] Papavasiliou, A., Pavliotis, G.A., Stuart, A. M. (2009) Maximum likelihood drift estimation for multi-scale diffusions. Stochastic Process. Appl., 119 (10), 3173-3210.

[21] Pavliotis, G.A., Stuart, A. M. (2007) Parameter estimation for multiscale diffusions. J. Stat. Phys., 127 (4), 741-781.

[22] Pavliotis, G.A., Stuart, A. M. (2008) Multiscale Methods. Texts in Applied Mathematics, Averaging and harmonization 53, Springer, New York

[23] Tikhonov. A.N. (1950) On system of differential equations containing parameters. Matem. Sbornik, 27 (69), 147-156.

[24] Tikhonov. A.N. (1952) System of differential equations containing a small parameters at derivatives. Matem. Sbornik, 31 (73), 147-156. 